\title{
Heterogeneously integrated InGaAsSb detectors on SOI waveguide circuits for short-wave infrared applications
}

\author{
N. Hattasan ${ }^{1}$, A. Gassenq ${ }^{1}$, B. Kuyken ${ }^{1}$, L. Cerutti ${ }^{2}$, J.B. Rodriguez ${ }^{2}$, E. Tournié ${ }^{2}$, D. Van Thourhout ${ }^{1}$, G. \\ Roelkens ${ }^{1}$ \\ ${ }^{1}$ Photonics Research Group, Ghent University - imec, Sint-Pietersnieuwstraat 41, 9000 Gent, Belgium \\ ${ }^{2}$ Université Montpellier 2 - CNRS, UMR 5214, Place Bataillon, 34095 Montpellier, France \\ Author e-mail address: nannicha.hattasan@intec.ugent.be
}

\begin{abstract}
We present evanescently coupled, heterogeneously integrated InGaAsSb photodetectors on silicon-on-insulator waveguide circuits for short-wave infrared applications. A fiber-to-detector responsivity of $0.13 \mathrm{~A} / \mathrm{W}$ is obtained at a wavelength of $2.17 \mu \mathrm{m}$. The photodetector dark current is $3.5 \mu \mathrm{A}$ at $-1 \mathrm{~V}$.

OCIS codes: (130.0130) Integrated optics; (130.3120) Integrated optics devices
\end{abstract}

\section{Introduction}

While silicon photonics is an important platform technology for applications in the telecommunication wavelength band, the use of silicon photonics in other wavelength ranges has been neglected so far. However, many applications could benefit from a silicon photonic integrated circuit for wavelengths outside the telecommunication wavelength range. Especially, towards longer wavelengths $(2-3.5 \mu \mathrm{m})$, silicon photonic circuits could become the dominant platform for integrated spectroscopic solutions, in which a short-wave infrared broadband beam probes an analyte which contains strong and very substance-specific absorption bands in this wavelength range [1]. Silicon photonic waveguide circuits are very appealing to realize such a spectroscopic function, given the transparency of the waveguide circuit in that wavelength range and the available know-how on how to realize compact integrated spectrometers on this platform using CMOS fabrication technology. In order to realize such an integrated spectroscopic system, the dense integration of short-wave infrared compatible photodetectors is required. While monolithically integrated Ge photodetectors are key components for the telecommunication wavelength range, their response cuts off at around $1.6 \mu \mathrm{m}$. In order to extend the wavelength range, the heterogeneous integration of III-V semiconductor photodetectors is required. There are two approaches to extend the operational wavelength range towards longer wavelengths. One approach is to use ternary InGaAs absorption layers grown on an InP substrate. While the cut-off wavelength of lattice matched InGaAs is only $1.7 \mu \mathrm{m}$ [2], further probing the short-wave infrared can be done by using lattice-mismatched "extended" InGaAs, which allows reaching the $2.5 \mu$ m wavelength range. However, in this work, we focus on an alternative approach, which relies on quaternary InGaAsSb absorbing layers in a p-i-n layer stack, grown lattice matched on a GaSb substrate. We will show that these photodetector structures can be heterogeneously integrated on and coupled to a silicon-on-insulator waveguide circuit, using an adhesive bonding technique based on DVS-BCB bonding. Experimentally, we obtain a fiber-to-detector responsivity of 0.13 $\mathrm{A} / \mathrm{W}$ at a wavelength of $2.17 \mu \mathrm{m}$, with a dark current of $3.5 \mu \mathrm{A}$ at $-1 \mathrm{~V}$.

\section{Device design}

The photodetectors are thin-film $\mathrm{p}-\mathrm{i}-\mathrm{n}$ photodetectors integrated on top of the silicon waveguide circuit using a DVS-BCB adhesive layer.

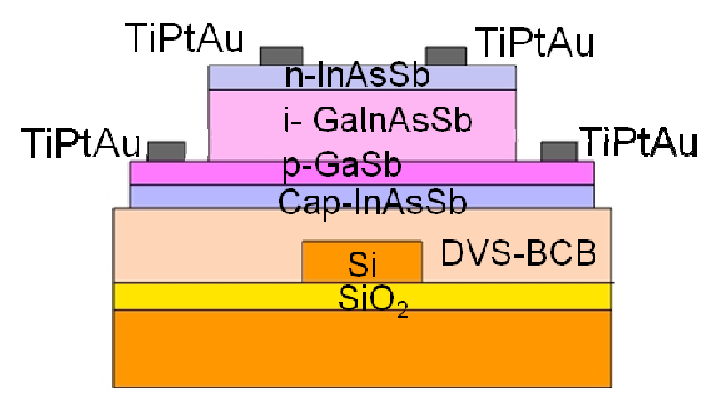

(a)

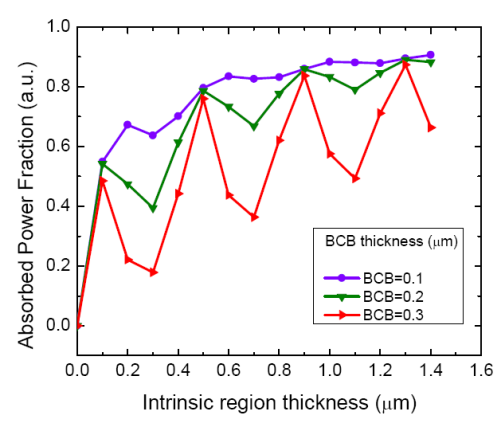

(b)

Figure 1. a) Cross-section of an evanescently coupled InGaAsSb photodetector based on BCB bonding; b) influence of the InGaAsSb absorbing layer thickness on the absorbed power fraction in a 50um long PD 
The interfacing between the silicon waveguide and the InGaAsSb photodetector is realized using evanescent coupling. A cross-section of the envisaged structure is shown in figure 1a. In this way a vertical directional coupler structure is realized which allows power transfer between the two waveguide layers. Optimization of the photodetector epitaxy stack thickness is required to realize an optimally compact photodetector, since the most efficient power transfer occurs when phase matching is achieved between the silicon waveguide mode and a guided mode in the III-V epitaxial layer stack. This is shown in figure $1 \mathrm{~b}$, showing the absorbed power fraction for a given detector stack $(50 \mu \mathrm{m}$ long) as a function of the intrinsic absorbing layer thickness and BCB bonding layer thickness.

\section{Device fabrication}

The silicon waveguide circuits used consist of a $220 \mathrm{~nm}$ silicon waveguide layer on a $2 \mu \mathrm{m}$ buried oxide layer. The waveguide losses of a single mode waveguide in the $2-2.5 \mu \mathrm{m}$ wavelength range are in the range of $3 \mathrm{~dB} / \mathrm{cm}$, as characterized by a cut-back measurement. The photodetector layer stack consists of an n-doped InAsSb cladding layer, an intrinsic InGaAsSb absorbing layer, a p-doped GaSb contact layer and an InAsSb cap layer, grown on a GaSb substrate using MBE [3]. While originally the cap-layer was to be removed prior to bonding, to get a clean semiconductor surface, this prevented good bonding due to the hydrophilic nature of the III-V surface after removal of the cap-layer. Therefore, this layer was incorporated in the photodetector layer stack. In order to integrate the III$\mathrm{V}$ epi-stack onto the silicon waveguide circuit, a die-to-wafer bonding process is applied, using DVS-BCB as a polymer bonding agent [4]. By diluting the DVS-BCB using mesitylene, bonding of GaSb-based epi-structures with bonding layer thicknesses down to $60 \mathrm{~nm}$ were successfully realized. After bonding the III-V die, epitaxial layers down onto the silicon waveguide circuit, the GaSb substrate is removed using a combination of mechanical grinding and wet chemical etching using a $\mathrm{HF}: \mathrm{CrO}_{3}$ solution, until an InAsSb etch stop layer is reached. High quality epitaxial films were obtained this way. After epitaxial layer transfer, photodetectors are processed, lithographically aligned to the underlying SOI waveguide circuit, as shown in figure $2 \mathrm{a}$. The mesa definition is realized using a combination of wet (Citric acid: $\left.\mathrm{H}_{2} \mathrm{O}_{2}: \mathrm{H}_{3} \mathrm{PO}_{4}: \mathrm{H}_{2} \mathrm{O}\right)$ and dry etching $\left(\mathrm{CH}_{4}: \mathrm{H}_{2}\right)$ in order to optimize the dark current of the resulting device. A cross-section of a fabricated photodetector is shown in figure $2 \mathrm{~b}$, clearly showing the undercut due to the wet chemical etching of the mesa. The mesa is $9 \mu \mathrm{m}$ wide.

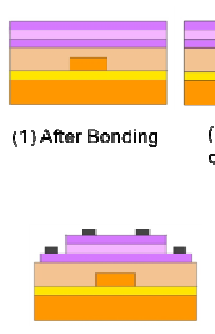

(5) Isla רd deinni:ion

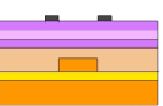
(2: II-cunlact
depssition

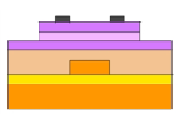

(3) Fo-ming mesa by (4) p-contect ICP $\left(\mathrm{CH}_{4}\right.$ arc $\left.\mathrm{H}_{2}\right)$ depusitior

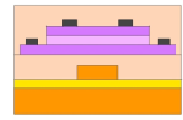

(0) BCB passivation

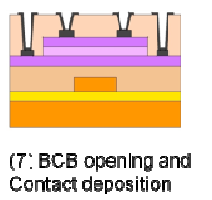

Contact deposition

(a)

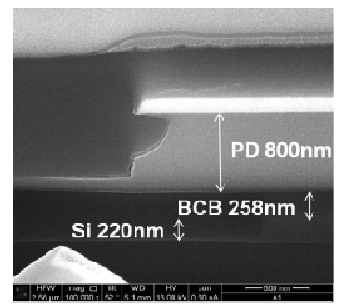

(b)

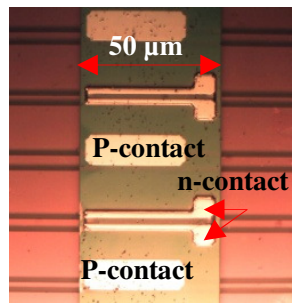

(c)

Figure 2. a) Schematic representation of process flow for the InGaAsSb photodetectors; b) focused ion beam cross-section image of the fabricated devices ; c) top-view of the fabricated photodetectors on top of a silicon waveguide circuit

\section{Device characterization}

The devices were characterized at room temperature. The setup consists of a mid-infrared tunable laser that is coupled to standard SMF. After polarization control, the light is injected into the silicon waveguide circuit using 
diffractive grating couplers defined in the silicon waveguide layer. Preliminary characterization of the grating coupler showed a peak fiber coupling efficiency of $-11 \mathrm{~dB}$ at a wavelength of $2.17 \mathrm{um}$ for TE polarized light. Since the integrated photodetector is only $600 \mu \mathrm{m}$ away from the grating coupler, the loss in the silicon entrance waveguide (due to scattering losses and the BCB absorption loss) can be neglected. Figure 3a shows the obtained IV curves for the fabricated devices under illumination (at $2170 \mathrm{~nm}$ ). A low dark current of $3.5 \mu \mathrm{A}$ at room temperature can be observed. A fiber-to-chip responsivity of $0.13 \mathrm{~A} / \mathrm{W}$ was realized, corresponding to an on-chip device responsivity of more than $1 \mathrm{~A} / \mathrm{W}$. High responsivities were obtained up to $2.4 \mu \mathrm{m}$. The linear response as a function of input power is shown in figure $3 \mathrm{~b}$. To our knowledge this is the first realization of an integrated photodetector capable of addressing the $2-2.5 \mu \mathrm{m}$ wavelength range.

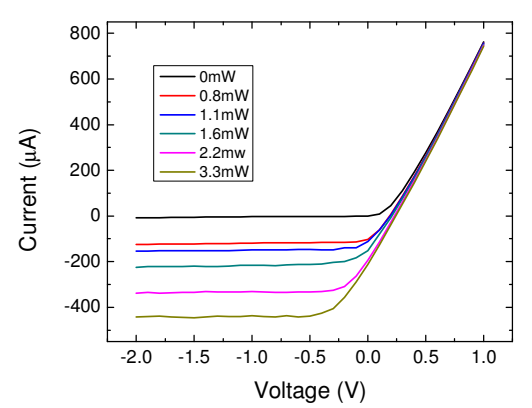

(a)

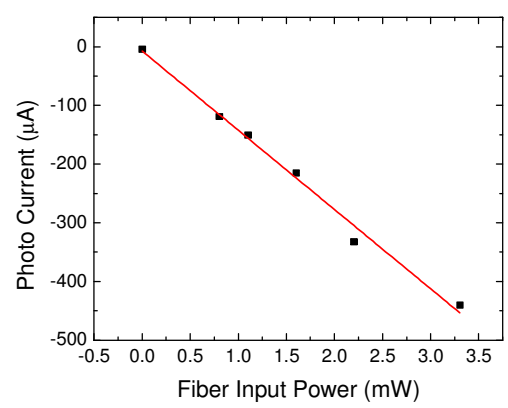

(b)

Figure 3. a) IV characteristics of the fabricated photodetectors showing a low dark current and good responsivity; b) linearity of the photodetector response

\section{Conclusions}

This paper describes the first realization of heterogeneously integrated InGaAsSb photodetectors on a silicon waveguide circuit. The device performance is very promising for the realization of integrated spectroscopic systems on silicon, provided that device arrays can be realized with good uniformity. Initial results, which will be discussed at the conference, show that this is the case. This opens many opportunities for the use of ultra-compact siliconbased spectroscopy systems, e.g. for the monitoring of glucose levels in blood samples or for gas sensing applications. The emerging field of non-linear optics beyond the two-photon absorption threshold in silicon [5], might also benefit from the availability of integrated photodetectors on the waveguide platform.

\section{Acknowledgements}

This work was partly carried out in the framework of the IWT-SBO-Glucosens project and the FP7-ERC-MIRACLE project.

\section{References}

[1] Crowder et al.,'Infrared methods for gas detection”, in Mid-infrared semiconductor optoelectronics, Springer (2006)

[2] J. Brouckaert, G. Roelkens, D. Van Thourhout, R. Baets, Compact InAlAs/InGaAs Metal-Semiconductor-Metal Photodetectors Integrated on Silicon-on-Insulator Waveguides, IEEE Photonics Technology Letters, 19(19), p.1484-1486 (2007)

[3] E. Tournié and A. Trampert, "MBE growth and interface formation of compound semiconductor heterostructures for optoelectronics," Phys. Stat. Sol. (b) 244 (8), 2683-2696 (2007)

[4] G. Roelkens, J. Brouckaert, D. Van Thourhout, R. Baets, R. Notzel, M. Smit, Adhesive Bonding of InP/InGaAsP Dies to Processed Siliconon-Insulator Wafers using DVS-bis-Benzocyclobutene,Journal of Electrochemical Society, 153(12), p.G1015-G1019 (2006)

[5] X. Liu, R.M. Osgood, Y. Vlasov, and W. Green, "Mid-infrared optical parametric amplifier using silicon nanophotonic waveguides", Nature Photonics 4, 557-560 (2010) 